\title{
Cold sensitivity of the SARS-CoV-2 spike ectodomain
}

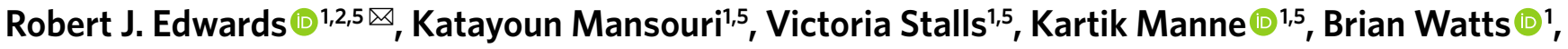 \\ Rob Parks', Katarzyna Janowska', Sophie M. C. Gobeil@ ', Megan Kopp', Dapeng Li', Xiaozhi Lu', \\ Zekun Mu๑1, Margaret Deyton', Thomas H. Oguin III', Jordan Sprenz', Wilton Williams ${ }^{1,2}$, \\ Kevin O. Saunders ${ }^{10}{ }^{1,3}$, David Montefiori ${ }^{1,3}$, Gregory D. Sempowski', Rory Henderson ${ }^{10}{ }^{1,2}$, \\ S. Munir Alam ${ }^{1,2}$, Barton F. Haynes ${ }^{1,2,4}$ and Priyamvada Acharya ${ }^{1,3 凶}$
}

\begin{abstract}
The SARS-CoV-2 spike (S) protein, a primary target for COVID-19 vaccine development, presents its receptor binding domain in two conformations, the receptor-accessible 'up' or receptor-inaccessible 'down' states. Here we report that the commonly used stabilized $S$ ectodomain construct ' $2 \mathrm{P}$ ' is sensitive to cold temperatures, and this cold sensitivity is abrogated in a 'down' state-stabilized ectodomain. Our findings will impact structural, functional and vaccine studies that use the SARS-CoV-2 S ectodomain.
\end{abstract}

The spike (S) protein of SARS-CoV-2 mediates receptor binding and cell entry and is a key target for vaccine development efforts. Stabilized S ectodomain constructs have been developed that mimic the native spike, bind the ACE- 2 receptor ${ }^{1,2}$ and present epitopes for neutralizing antibodies on their surface ${ }^{3-6}$. The so-called ' 2 P'S ectodomain construct (2PS) comprises residues 1-1,208 of SARS-CoV-2 S and contains two proline (2P) substitutions in the $\mathrm{C}$-terminal S2 domain designed to stabilize the prefusion $\mathrm{S}$ conformation; a C-terminal foldon trimerization motif and a mutation that abrogates the furin-cleavage site $^{1}$ (Fig. 1a). This and similar constructs have been widely used for structural biology and vaccine studies $^{1-3,7,8}$. Purified S ectodomain proteins ${ }^{9}$ are assessed for quality control by SDS-PAGE, size exclusion chromatography (SEC), differential scanning fluorimetry (DSF) ${ }^{10}$ and negative-stain electron microscopy (NSEM). The last technique has been particularly informative because it reveals the structural integrity of individual molecules, allowing us to examine preparations that look similar by using bulk methods such as SDS-PAGE and SEC (Supplementary Fig. 1). The observed variability between preparations indicates a fragile $S$ ectodomain, and measures to overcome the issue have been previously reported ${ }^{11,12}$.

Here we link the apparent fragility of 2PS to its rapid denaturation on storage at $4^{\circ} \mathrm{C}$ (Fig. 1b). We followed the structural, biophysical and antigenic properties of 2PS stored under different temperature conditions (Fig. 1, Extended Data Figs. 1 and 2, Supplementary Tables 1 and 2 and Supplementary Figs. 2-6). 2PS was produced in $293 \mathrm{~F}$ cells at $37^{\circ} \mathrm{C}$ and purified at room temperature within $6-8 \mathrm{~h}$ (Supplementary Fig. 1). We performed NSEM analysis of 2PS incubated at different temperatures (Fig. 1b and Extended Data Fig. 1d,e). Freshly prepared 2P S samples assessed on the same day they were purified showed on average $75 \%$ well-formed spikes, with characteristic kite-shaped morphology on NSEM micrographs and two-dimensional (2D) class averages. This fraction slightly decreased to $64 \%$ after one cycle of freeze-thaw and to $59 \%$ after room temperature $\left(22^{\circ} \mathrm{C}\right)$ storage for $5-7$ days, and it was substantially reduced to $5 \%$ after storage at $4{ }^{\circ} \mathrm{C}$ for $5-7$ days. In contrast, after 1 week of storage at $37^{\circ} \mathrm{C}$, we observed $83 \%$ well-formed spikes. Furthermore, the well-formed spikes could be recovered after storage at $4{ }^{\circ} \mathrm{C}$ for 1 week with a 3 -h incubation at $37^{\circ} \mathrm{C}$ to roughly $75 \%$, and three-dimensional (3D) classification of those particles showed the typical populations of the all-receptor-binding domain- (RBD) down and 1-RBD-up spike that we reported previously for freshly prepared samples ${ }^{9}$ (Supplementary Fig. 6). No further recovery from $4{ }^{\circ} \mathrm{C}$ storage was observed after longer incubations at $37^{\circ} \mathrm{C}$, which actually led to slight aggregation (Extended Data Fig. 1e).

SDS-PAGE analysis of 2PS samples stored at different temperatures did not indicate any appreciable protein degradation, although we did observe an increase in higher molecular weight bands for samples stored at $37^{\circ} \mathrm{C}$ for 1 week (Supplementary Fig. 3). Similar to our NSEM results, we saw substantial differences in the quality and dispersion of particles in cryo-EM grids depending on the storage temperature of the specimen (Supplementary Fig. 4).

The $S$ ectodomain was reported to exhibit conformational changes in response to $\mathrm{pH}^{12}$, so we asked whether the $2 \mathrm{PS}$ denaturation we observed was due to temperature-dependent $\mathrm{pH}$ changes in the Tris $\mathrm{pH} 8.0$ buffer used $^{13}$. A Tris buffer solution that measures $\mathrm{pH} 8.0$ at $25^{\circ} \mathrm{C}$ would measure roughly $\mathrm{pH} 8.6$ at $4{ }^{\circ} \mathrm{C}$. We performed SEC purification of a $2 \mathrm{PS}$ preparation into 3-morpholinopropane-1-sulfonic acid (MOPS) buffer pH 7.4; with smaller temperature dependence compared to Tris ${ }^{13}$, MOPS buffer should change only slightly, to $\mathrm{pH} 7.42$, at $4^{\circ} \mathrm{C}$. Cold storage at $\mathrm{pH} 7.42$ (MOPS buffer) reduced the spike fraction to $4 \%$, similar to the average of $5 \%$ for cold storage in Tris buffer (Fig. 1b). Thus, temperature change appears to be the primary cause of $2 \mathrm{PS}$ denaturation, and not the $\mathrm{pH}$ shift within this range. We also tested the temperature effect at acidic $\mathrm{pH}$ and found that incubating $2 \mathrm{PS}$ at $4^{\circ} \mathrm{C}$ in MES, pH 6 buffer reduced but did not eliminate the cold sensitivity.

Next, we examined whether the observed cold sensitivity is a property of the bulk protein in solution rather than an artifact of the NSEM sample preparation. First, we used a rapid DSF assay ${ }^{10}$ that measures changes in the protein intrinsic fluorescence as a thermal ramp is applied. The changes in fluorescence signal indicate transitions in the folding state of a protein, and the temperature at which

'Duke Human Vaccine Institute, Durham, NC, USA. ${ }^{2}$ Department of Medicine, Duke University, Durham, NC, USA. ${ }^{3}$ Department of Surgery, Duke University, Durham, NC, USA. ${ }^{4}$ Department of Immunology, Duke University, Durham, NC, USA. ${ }^{5}$ These authors contributed equally: Robert J. Edwards, Katayoun Mansouri, Victoria Stalls, Kartik Manne.凶e-mail: rj.edwards@duke.edu; priyamvada.acharya@duke.edu 
a

SARS-CoV-2 S

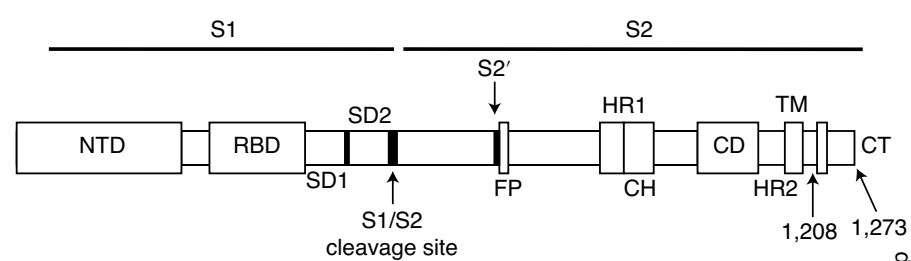

Stabilized $S$ ectodomain (2P S)

b
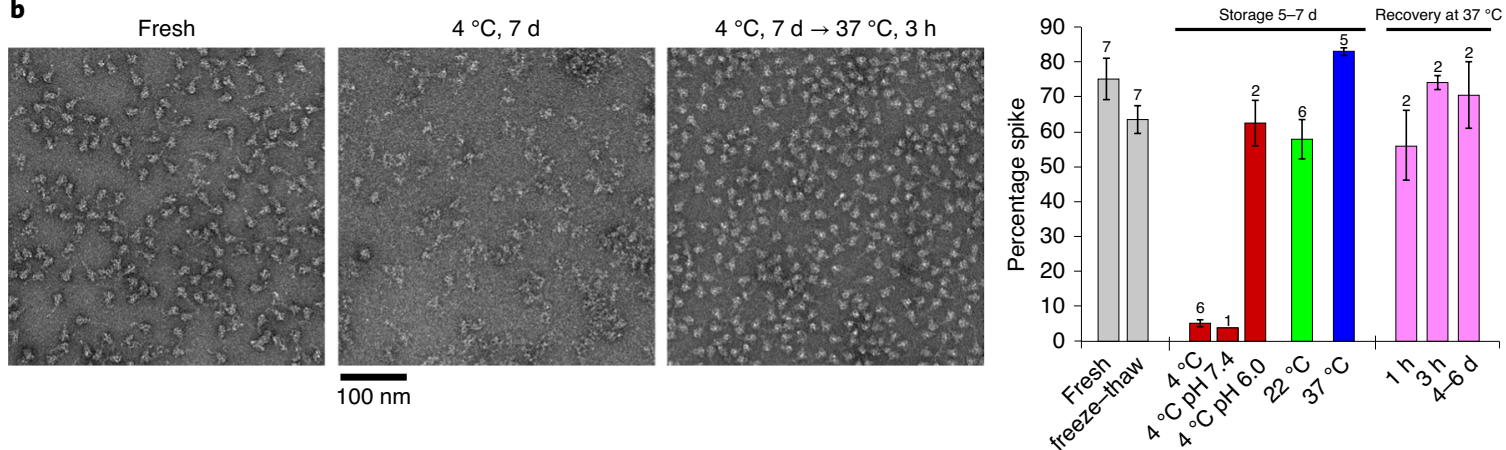

c
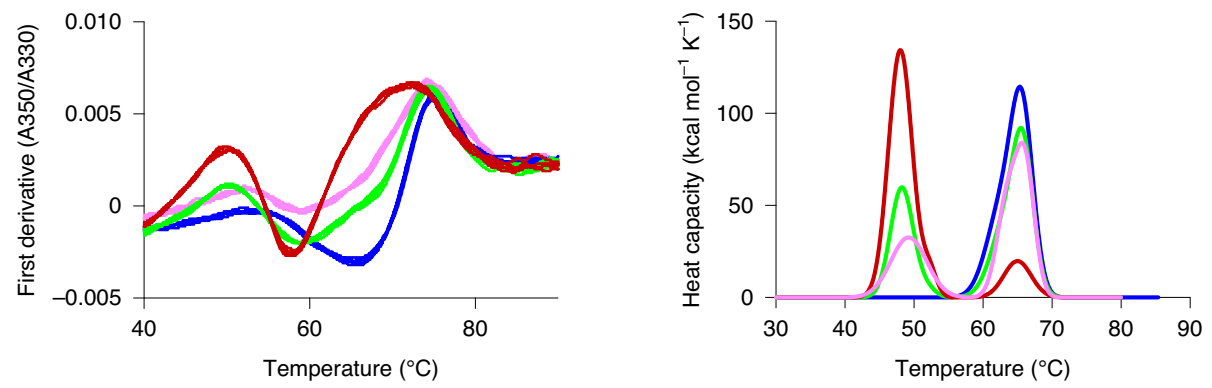

d
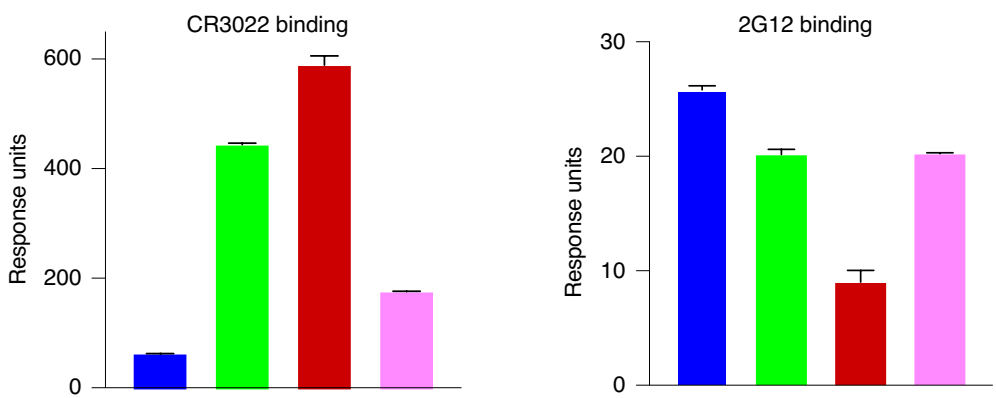

$37^{\circ} \mathrm{C}, 7 \mathrm{~d}$

$22^{\circ} \mathrm{C}, 7 \mathrm{~d}$

$4{ }^{\circ} \mathrm{C}, 7 \mathrm{~d}$

$4^{\circ} \mathrm{C}, 7 \mathrm{~d} \rightarrow 37^{\circ} \mathrm{C}$

Fig. 1 | Temperature dependence of the SARS-CoV-2 S ectodomain. a, Schematic of the SARS-CoV-2 spike (top, S) and a stabilized,

furin-cleavage-deficient, soluble ectodomain construct (bottom, 2P S). b, Representative NSEM micrographs of 2PS samples: freshly prepared (left), after storage at $4{ }^{\circ} \mathrm{C}$ for $7 \mathrm{~d}$ (middle) or stored at $4^{\circ} \mathrm{C}$ for $7 \mathrm{~d}$ followed by a 3 -h incubation at $37^{\circ} \mathrm{C}$ (right). Bar graph summarizes the results from NSEM analyses of 2PS samples stored under different conditions. Data shown are mean and range (for $n=2$ ) or mean and s.e.m. (from $n=3-7)$ independent experiments with different protein lots; exact $n$ at the top of each bar. c, Left, DSF profiles following changes in protein intrinsic fluorescence (expressed as a ratio between fluorescence at 350 and $330 \mathrm{~nm}$ ) on applying a thermal ramp. Maxima and minima indicate inflection temperatures, $T_{i}$. For each storage condition (color coded as shown in d), five overlaid curves (technical replicates) are shown. Right, DSC profiles, shown as a representative curve of two technical replicates. d, Antibody binding to 2PS stored at different temperatures, measured by SPR; antibodies were CR3022 lgG (left) and $2 \mathrm{G} 12$ lgG (right). During the SPR run, the sample chamber was maintained at 37,22 or $8^{\circ} \mathrm{C}$ for samples that had been stored at 37,22 or $4{ }^{\circ} \mathrm{C}$, respectively. The binding experiments were carried out at $25^{\circ} \mathrm{C}$. Data are mean and s.e.m. of three technical replicates and are representative of at least five independent experiments, using separate protein lots (two independent repeats are shown in Extended Data Fig. 2).

a transition occurs is called the inflection temperature $\left(T_{\mathrm{i}}\right)$. Distinct profile shifts were observed for $2 \mathrm{PS}$ samples stored at different temperatures, with $T_{\mathrm{i}}$ shifted toward lower temperatures for samples stored at $4{ }^{\circ} \mathrm{C}$, indicating lower protein stability, compared to samples stored at 22 or $37^{\circ} \mathrm{C}$ (Fig. 1c and Supplementary Table 1). Next, we measured melting temperatures $\left(T_{\mathrm{m}}\right)$ using differential scanning cal- 
a

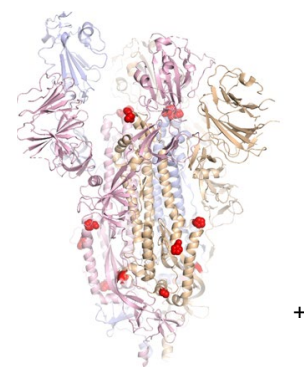

HexaPro (PDB ID 6XKL)

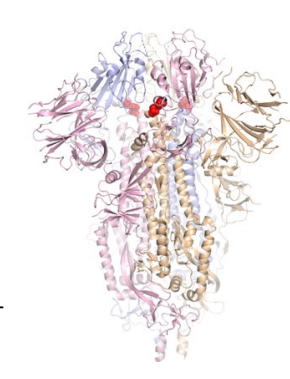

rS2d
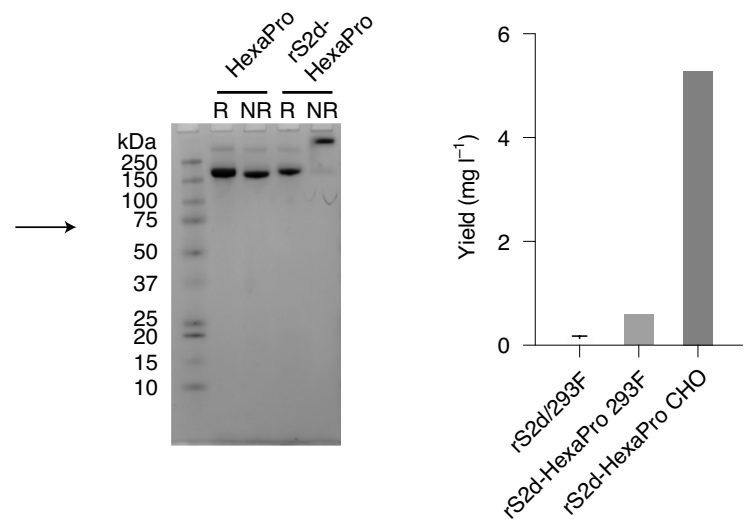

b
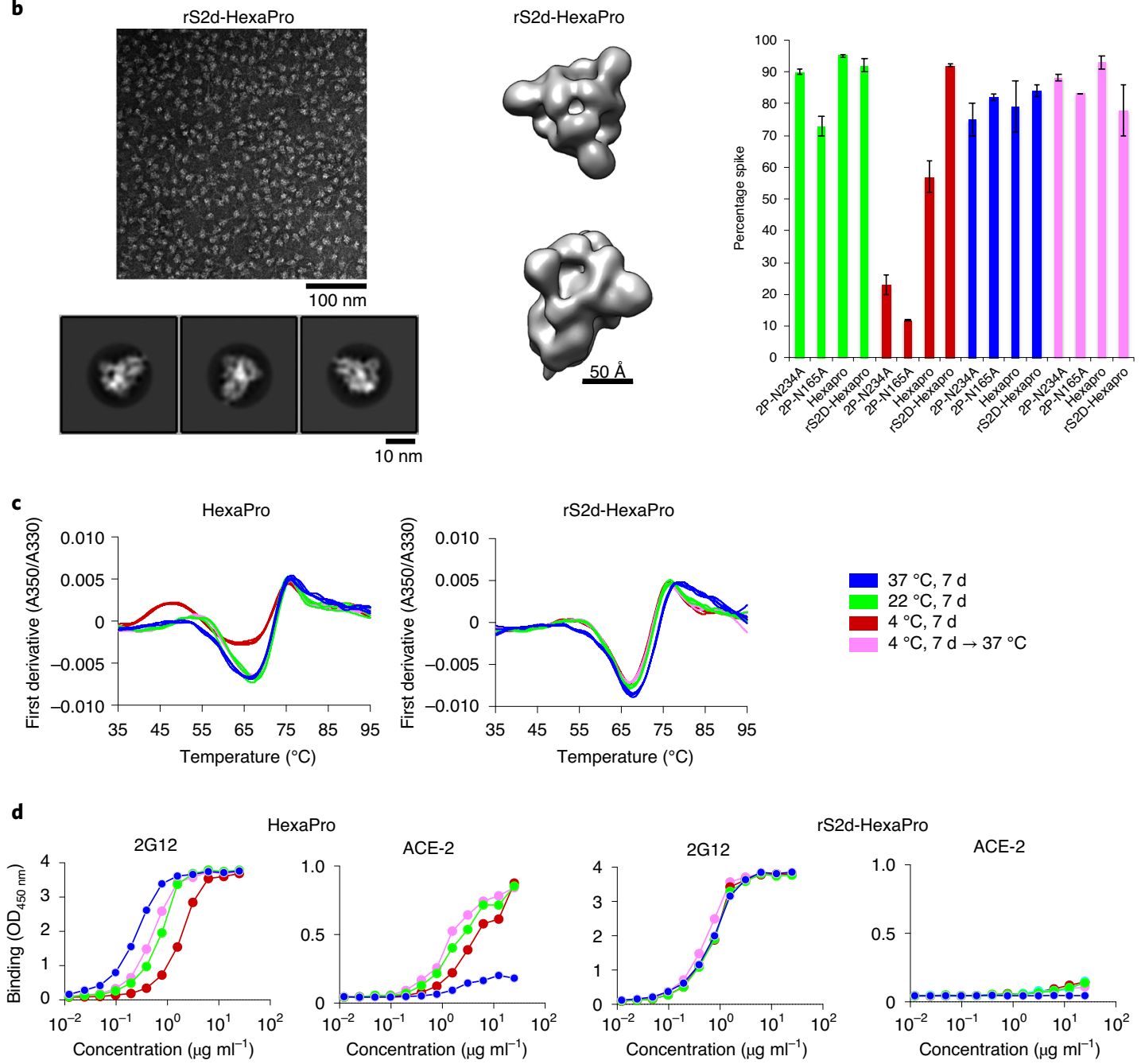

S2d-HexaPro

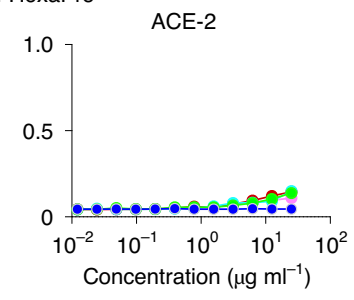

Fig. 2 | Engineered SARS-CoV-2 spike variant, rS2d-HexaPro, is resistant to temperature-dependent structural changes. a, Left, structures of HexaPro showing a 1-RBD-up conformation (PDB 6XKL) and rS2d (PDB 6X29) showing an all-RBD-down conformation. Middle, SDS-PAGE analysis of HexaPro and rS2d-HexaPro samples under reducing (R) or non-reducing (NR) conditions. Right, bar graph showing protein yields for rS2d and rS2d-HexaPro produced in $293 \mathrm{~F}$ or $\mathrm{CHO}$ cells. b, Left, representative NSEM micrograph from a preparation of rS2d-HexaPro (top) and 2D class averages (bottom). Middle, 3D reconstruction of rS2d-HexaPro. Right, bar graph summarizing results from NSEM on S ectodomain variants stored at different temperatures. Data are mean and range (for $n=2$ ) or s.e.m. (for $n \geq 3$ ) for independent experiments with different protein lots. c, DSF profiles for HexaPro (left) and rS2d-HexaPro (right). For each storage condition, 3-4 overlaid curves (technical replicates) are shown. d, Binding of $2 \mathrm{G} 12$ and ACE-2 to HexaPro (left) and rS2d-HexaPro (right) measured by ELISA. OD ${ }_{450 \mathrm{~nm}}$ optical density at $450 \mathrm{~nm}$.

orimetry (DSC) (Fig. 1c, Supplementary Table 1 and Supplementary Fig. 5); after 1 week of storage at $37^{\circ} \mathrm{C}$, the $2 \mathrm{PS}$ sample featured an asymmetric unfolding transition with $T_{\mathrm{m}}$ of $65.5^{\circ} \mathrm{C}$. After 1 week of storage at $22^{\circ} \mathrm{C}$, we observed a second low- $T_{\mathrm{m}}$ transition at $48.2^{\circ} \mathrm{C}$. After 1 week of storage at $4^{\circ} \mathrm{C}$, we observed a similar two-peak profile with a markedly more pronounced low- $T_{\mathrm{m}}$ transition 
$\left(T_{\mathrm{m}}=48.4^{\circ} \mathrm{C}\right)$. On returning the $4^{\circ} \mathrm{C}$ sample to $37^{\circ} \mathrm{C}$ for $3 \mathrm{~h}$ before analysis, we observed an amplitude reduction of the low- $T_{\mathrm{m}}$ transition $\left(T_{\mathrm{m}}=49.2^{\circ} \mathrm{C}\right)$ and a corresponding amplitude increase in the high- $T_{\mathrm{m}}$ transition $\left(T_{\mathrm{m}}=66.0^{\circ} \mathrm{C}\right)$. Thus, the DSC results confirm that storage at $4^{\circ} \mathrm{C}$ destabilizes $2 \mathrm{PS}$ compared to samples stored at 22 or $37^{\circ} \mathrm{C}$, and that returning the destabilized $2 \mathrm{PS}$ to $37^{\circ} \mathrm{C}$ for $3 \mathrm{~h}$ substantially restores its stability, although the presence of the low- $T_{\mathrm{m}}$ peak suggests that the recovery is partial.

We next tested the effects of cold-induced instability on ligand binding to 2PS using surface plasmon resonance (SPR) and enzyme-linked immunosorbent assay (ELISA) (Fig. 1d and Extended Data Figs. 2 and 3). We found that 2PS stored at $4{ }^{\circ} \mathrm{C}$ showed higher binding to antibody CR3022 and to receptor ACE-2, which both require an 'up' RBD conformation for stable binding ${ }^{1,9,12}$, compared to freshly prepared protein. In contrast, cold storage reduced binding to antibody $2 \mathrm{G} 12$, which recognizes a quaternary glycan epitope in the S2 subunit ${ }^{14}$, indicating loss of quaternary structure during cold storage. We also tested two antibodies isolated from a COVID-19 convalescent donor, with epitopes mapped to the ACE-2 binding site (DH1179) and to the S2 region of spike (DH1189.1) (Extended Data Fig. 3 and Supplementary Figs. 7 and 8). Both antibodies showed different binding profiles depending on the temperature at which 2PS was stored. We next tested CR3022 and 2 G12 binding to a $2 \mathrm{PS}$ sample that was flash frozen in liquid $\mathrm{N}_{2}$, stored in single-use aliquots at $-80^{\circ} \mathrm{C}$ and thawed by incubation at $37^{\circ} \mathrm{C}$ (Supplementary Fig. 9). Freeze-thaw increased CR3022 binding to the $2 \mathrm{PS}$ compared to fresh sample, but incubating the thawed sample at $37^{\circ} \mathrm{C}$ for $20 \mathrm{~min}$ reversed the effect, with binding activity similar to the levels observed with fresh sample.

These results indicated that cold-induced denaturation of the spike was associated with increased RBD-exposure of the $S$ ectodomain. We thus asked whether a 'down' state-stabilized $S$ ectodomain might be resistant to cold-induced denaturation (Fig. 2). Here we included a variant that combined the previously described rS2 $\mathrm{d}^{9}$ and HexaPro mutations ${ }^{11}$ (Fig. 2a). This new variant, named rS2d-HexaPro, showed higher production yields compared to $\mathrm{rS}_{2} \mathrm{~d}^{9}$. Similarly to $\mathrm{rS}^{2} \mathrm{~d}^{9}$, rS2d-HexaPro particles appear $100 \%$ in a 3 -RBD-down conformation as seen by NSEM (Fig. 2b, Supplementary Fig. 10 and Supplementary Video 1). Both HexaPro and rS2d-HexaPro appeared more resistant to cold-induced denaturation than 2P S (Fig. 1b), with rS2d-HexaPro showing a higher intact spike percentage than HexaPro (Fig. 2b). Thermostability and binding studies (Fig. 2c,d) further confirmed that HexaPro and rS2d-HexaPro were more resistant to cold destabilization compared to 2P S (Fig. 1).Two glycan-deleted mutants, $2 \mathrm{P}-\mathrm{N} 165 \mathrm{~A}$ and $2 \mathrm{P}-\mathrm{N} 234 \mathrm{~A}^{15}$, which have altered RBD-up propensity, showed substantial reduction of intact spike after incubation at $4{ }^{\circ} \mathrm{C}$ (Fig. 2f). The rS2d mutations (D985C, S383C) introduce an inter-protomer disulfide bond between the RBD and the S2 domain. Another study confirmed the stabilization effect of this disulfide bond, as well as that of an introduced disulfide bond between residues 987 and 413 , for spikes stored at $4^{\circ} \mathrm{C}$ (ref. ${ }^{16}$ ). These data indicate that inter-promoter disulfide linkages may be a general strategy to stabilize the $2 \mathrm{P}$ S ectodomain and prevent its cold-induced denaturation.

Compared to 2PS, rS2d-HexaPro binding to ACE-2 was substantially reduced for samples stored at all temperatures, as expected for a spike fixed in an RBD-down conformation. Similarly, binding to $2 \mathrm{G} 12$ remained same for the rS2d-HexaPro construct irrespective of the storage temperature. For HexaPro, binding to ACE-2 was higher and $2 \mathrm{G} 12$ binding was reduced with $4{ }^{\circ} \mathrm{C}$-stored protein compared to $37^{\circ} \mathrm{C}$-stored samples, showing that the HexaPro structure remained susceptible to perturbation at lower temperatures, whereas rS2d-HexaPro remains unperturbed on $4^{\circ} \mathrm{C}$ storage.

Overall, our results demonstrate cold sensitivity of the furin-cleavage-deficient SARS-CoV-2 S ectodomain and highlight the importance of accounting for this effect, to obtain consistent results in serology assays or other binding studies using the $2 \mathrm{PS}$ or similar constructs. Based on these data, we recommend flash freezing spike ectodomain samples in single-use aliquots, then thawing at $37^{\circ} \mathrm{C}$ followed by a brief (roughly $20 \mathrm{~min}$ ) incubation at $37^{\circ} \mathrm{C}$ before use in binding studies.

\section{Online content}

Any methods, additional references, Nature Research reporting summaries, source data, extended data, supplementary information, acknowledgements, peer review information; details of author contributions and competing interests; and statements of data and code availability are available at https://doi.org/10.1038/ s41594-020-00547-5.

Received: 29 July 2020; Accepted: 3 December 2020; Published online: 5 January 2021

\section{References}

1. Wrapp, D. et al. Cryo-EM structure of the 2019-nCoV spike in the prefusion conformation. Science 367, 1260-1263 (2020).

2. Walls, A. C. et al. Structure, function, and antigenicity of the SARS-CoV-2 spike glycoprotein. Cell 181, 281-292.e6 (2020).

3. Barnes, C. O. et al. Structures of human antibodies bound to SARS-CoV-2 spike reveal common epitopes and recurrent features of antibodies. Cell 182, 828-842.e16 (2020).

4. Pinto, D. et al. Cross-neutralization of SARS-CoV-2 by a human monoclonal SARS-CoV antibody. Nature 583, 290-295 (2020).

5. Mire, C. E. et al. A cross-reactive humanized monoclonal antibody targeting fusion glycoprotein function protects ferrets against lethal Nipah virus and hendra virus infection. J. Infect. Dis. 221, S471-S479 (2020).

6. Ke, Z. et al. Structures, conformations and distributions of SARS-CoV-2 spike protein trimers on intact virions. Nature 588, 498-502 (2020).

7. Liu, L. et al. Potent neutralizing monoclonal antibodies directed to multiple epitopes on the SARS-CoV-2 Spike. Nature 584, 450-456 (2020).

8. Zhou, T. et al. Structure-based design with tag-based purification and in-process biotinylation enable streamlined development of SARS-CoV-2 spike molecular probes. Cell Reports 33, https://doi.org/10.1016/j. celrep.2020.108322 (2020).

9. Henderson, R. et al. Controlling the SARS-CoV-2 spike glycoprotein conformation. Nat. Struct. Mol. Biol. 27, 925-933 (2020).

10. Magnusson, A. O. et al. nanoDSF as screening tool for enzyme libraries and biotechnology development. FEBS J. 286, 184-204 (2019).

11. Hsieh, L. et al. Structure-based design of prefusion-stabilized SARS-CoV-2 spikes. Science 369, 1501-1505 (2020).

12. Zhou, T. et al. Cryo-EM structures of SARS-CoV-2 spike without and with ACE2 reveal a $\mathrm{pH}$-dependent switch to mediate endosomal positioning of receptor-binding domains Cell Host Microbe https://doi.org/10.1016/j. chom.2020.11.004 (2020).

13. Good, N. E. et al. Hydrogen ion buffers for biological research. Biochemistry 5, 467-477 (1966).

14. Acharya, P. et al. A glycan cluster on the SARS-CoV-2 spike ectodomain is recognized by Fab-dimerized glycan-reactive antibodies. Preprint bioRxiv https://doi.org/10.1101/2020.06.30.178897 (2020).

15. Henderson, R. et al. Glycans on the SARS-CoV-2 spike control the receptor binding domain conformation. Preprint at bioRxiv https://doi. org/10.1101/2020.06.26.173765 (2020).

16. Xiong, X. et al. A thermostable, closed SARS-CoV-2 spike protein trimer. Nat. Struct. Mol. Biol. 27, 934-941 (2020).

Publisher's note Springer Nature remains neutral with regard to jurisdictional claims in published maps and institutional affiliations.

(c) The Author(s), under exclusive licence to Springer Nature America, Inc. 2021 


\section{Methods}

Protein expression and purification. SARS-CoV-2 ectodomain constructs were produced and purified as described previously ${ }^{1,11}$. Briefly, a gene encoding residues $1-1,208$ of the SARS-CoV-2 S (GenBank MN908947) with proline substitutions at residues 986 and 987, a 'GSAS' substitution at the furin-cleavage site (residues 682-685), a C-terminal T4 fibritin trimerization motif, an HRV3C protease cleavage site, a TwinStrepTag and an $8 \times$ HisTag was synthesized and cloned into the mammalian expression vector $\mathrm{p} \alpha \mathrm{H}$ (Fig. 1a). Plasmids were transiently transfected into FreeStyle-293F cells using Turbo293 (SpeedBiosystems). Cells were grown in a $37^{\circ} \mathrm{C}$ incubator with $9 \% \mathrm{CO}_{2}$ and 120 r.p.m. rotary motion. Protein was purified on the sixth day post transfection from filtered supernatant using StrepTactin resin (IBA), followed by SEC purification using a Superose $610 / 300$ Increase column in $\mathrm{nCoV}$ buffer $(2 \mathrm{mM}$ Tris, $\mathrm{pH} 8.0,200 \mathrm{mM} \mathrm{NaCl}, 0.02 \%$ sodium azide). rS2d-HexaPro was also grown in CHO cells. Plasmids were transiently transfected into CHO cells using ExpiFectamine CHO Transfection Kit (Thermo Fisher), and protein was purified on the sixth day post transfection. All protein purification steps including Strep-tag purification and SEC were performed at room temperature. The spikes were purified the same day that the supernatant was harvested, and the purification was completed within $6-8 \mathrm{~h}$. The purified protein was flash frozen and stored at $-80^{\circ} \mathrm{C}$ in single-use aliquots. Each aliquot was thawed and briefly incubated (roughly $20 \mathrm{~min}$ ) at $37^{\circ} \mathrm{C}$ before use.

Antibodies were produced in Expi293 cells and purified by Protein A affinity. For ACE- 2 constructs, the ACE- $2 \mathrm{C}$ terminus was fused with either the human or mouse Fc region including a C-terminal $6 \times$ His-tag on the Fc domain. ACE- 2 with human Fc tag was purified by Protein A affinity chromatography, and ACE- 2 with mouse Fc tag was purified by Ni-NTA chromatography.

NSEM. Spike samples were incubated for the specified times in nCoV buffer at 4,22 or $37^{\circ} \mathrm{C}$, then moved to room temperature to prepare NSEM grids, which was complete in less than $5 \mathrm{~min}$. Samples were diluted to $100 \mu \mathrm{g} \mathrm{ml}^{-1}$ with room-temperature buffer containing $20 \mathrm{mM}$ HEPES pH 7.4, $150 \mathrm{mM} \mathrm{NaCl}, 5 \%$ glycerol and $7.5 \mathrm{mM}$ glutaraldehyde, and incubated for $5 \mathrm{~min}$; then glutaraldehyde was quenched for $5 \mathrm{~min}$ by addition of $1 \mathrm{M}$ Tris stock to a final concentration of $75 \mathrm{mM}$. A 5- $\mu$ l drop of sample was applied to a glow-discharged, carbon-coated grid for $10-15 \mathrm{~s}$, blotted, stained with $2 \%$ uranyl formate, blotted and air dried. Images were obtained with a Philips EM420 electron microscope at $120 \mathrm{kV}$, $\times 82,000$ magnification and a $4.02 \AA$ pixel size. The RELION program ${ }^{17}$ was used for particle picking, and $2 \mathrm{D}$ and $3 \mathrm{D}$ class averaging.

Thermostability assays. Thermostability of the $\mathrm{S}$ ectodomain samples was measured using DSF and DSC. Samples were purified and buffer exchanged into HEPES buffered saline (HBS) buffer (10 mM HEPES, $150 \mathrm{mM} \mathrm{NaCl}, \mathrm{pH} 7.4$ ) by SEC on a Superose 6 10/300 column. DSF assay was performed using Tycho NT. 6 (NanoTemper Technologies). Spike variants were diluted $\left(0.15 \mathrm{mg} \mathrm{ml}^{-1}\right)$ in HBS and run in triplicate. Intrinsic fluorescence was recorded at 330 and $350 \mathrm{~nm}$ while heating the sample from $35-95^{\circ} \mathrm{C}$ at a rate of $30^{\circ} \mathrm{C} \mathrm{min}{ }^{-1}$. The ratio of fluorescence $(350 / 330 \mathrm{~nm})$ and inflection temperatures $\left(T_{\mathrm{i}}\right)$ were calculated by Tycho NT. 6 .

DSC measurements were performed using the NanoDSC platform (TA Instruments). Samples that had been incubated at 22 or $37^{\circ} \mathrm{C}$ were purified by SEC at room temperature and the sample incubated at $4^{\circ} \mathrm{C}$ was purified by SEC at $4^{\circ} \mathrm{C}$ (Extended Data Fig. 2), diluted to $0.2-0.3 \mathrm{mg} \mathrm{ml}^{-1}$ in HBS, and degassed for $15 \mathrm{~min}$ at room temperature before analysis. DSC measurements were performed immediately after SEC purification of the samples. DSC cells were conditioned with filtered, degassed HBS before sample loading. Protein samples were heated from $10^{\circ} \mathrm{C}$ to $100^{\circ} \mathrm{C}$ at $1^{\circ} \mathrm{C} \mathrm{min}^{-1}$ under 3 atm pressure using HBS as the reference buffer. The observed denaturation profiles were buffer subtracted, converted to molar heat capacity, baseline-corrected with a sixth-order polynomial and fit with 2-4 Gaussian transition models, as needed, using the NanoAnalyze software (TA Instruments). The peak transition temperature $\left(T_{\mathrm{m}}\right)$ is reported as the temperature at the maximum observed heat capacity of each transition peak.

Isolation of antibodies from COVID-19 convalescent donors. Human SARS-CoV-2 spike antibodies DH1179 and DH1189.1 were isolated from a COVID-19 convalescent individual. Peripheral blood was collected following informed consent on a Duke University Medical Center approved Institutional Review Board protocol. Briefly, peripheral blood mononuclear cell samples collected after the onset of the symptoms were stained and the memory B cells were sorted with SARS-CoV-2 $2 \mathrm{P}$ S probes. Antibody $I g H$ and $I g K / L$ genes were recovered from the single-cell sorted cells, cloned into human IgG1 constant region backbone and purified by Protein A beads as previously described ${ }^{18}$.

ELISA assays. Spike samples were preincubated at different temperatures then tested for antibody- or ACE-2-binding in ELISA assays as previously described ${ }^{19,20}$. Assays were run in two formats. In the first format, antibodies or ACE-2 protein were coated on 384 -well plates at $2 \mu \mathrm{g} \mathrm{ml}^{-1}$ overnight at $4^{\circ} \mathrm{C}$, washed, blocked and followed by twofold serially diluted spike protein starting at $25 \mu \mathrm{g} \mathrm{ml}^{-1}$. Binding was detected with polyclonal anti-SARS-CoV-2 spike rabbit serum (developed in our laboratory), followed by goat anti-rabbit-HRP (Abcam no. ab97080) and 3,3',5,5' -tetramethylbenzidine (TMB) substrate (Sera Care Life Sciences no. 5120-0083). Absorbance was read at $450 \mathrm{~nm}$. In the second format, serially diluted spike protein was bound in individual wells of 384-well plates, which were previously coated with streptavidin (Thermo Fisher Scientific no. S-888) at $2 \mu \mathrm{g} \mathrm{ml}^{-1}$ and blocked. Proteins were incubated at room temperature for $1 \mathrm{~h}$, washed, then human mAbs were added at $10 \mu \mathrm{g} \mathrm{ml}^{-1}$. Antibodies were incubated at room temperature for $1 \mathrm{~h}$, washed and binding detected with goat anti-human-HRP (Jackson ImmunoResearch Laboratories, no. 109-035-098) and TMB substrate.

Commercially obtained constructs of SARS-CoV-2 spike ectodomain (S1+S2 ECD, S2 ECD and RBD) (Sino Biological Inc. catalog no. 40589-V08B1 and 40590-V08B, respectively, and RBD from Genescript catalog no. Z03483) were coated directly on 384-well plates at $2 \mu \mathrm{g} \mathrm{ml}^{-1}$ and incubated overnight at $4{ }^{\circ} \mathrm{C}$. Plates were washed and blocked, and human mAbs threefold serially diluted from $100 \mu \mathrm{g} \mathrm{ml}^{-1}$ were added for $1 \mathrm{~h}$ at room temperature followed by washing. Binding was detected with goat anti-human IgG-HRP followed by TMB substrate.

Microneutralization assay. Live SARS-CoV-2 microneutralization assays were adapted from Berry et al..$^{21}$ In short, sera or purified antibodies are diluted twofold and incubated with 100 median tissue culture infectious dose $\left(\mathrm{TCID}_{50}\right)$ virus for $1 \mathrm{~h}$. These dilutions are used as the input material for a $\mathrm{TCID}_{50}$. Each batch of microneutralization includes known neutralizing and non-neutralizing controls. Data are reported as the last concentration at which a test sample protects Vero E6 cells. Recently, a fluorescent infectious clone of SARS-CoV-2 was developed ${ }^{22}$.

Plaque reduction neutralization test (PRNT). A live SARS-CoV-2 PRNT was performed in an assay adapted from Berry et al. ${ }^{21}$ Briefly, twofold dilutions of a test sample (serum, plasma, purified $\mathrm{Ab}$ ) are incubated with 50 plaque forming units of SARS-CoV-2 for $1 \mathrm{~h}$ (NR-52281, BEI Resources). The antibody/virus mixture was then used to inoculate Vero E6 cells (ATCC). Cultures were then incubated at $37^{\circ} \mathrm{C}, 5 \% \mathrm{CO}_{2}$ for $1 \mathrm{~h}$. At the end of the incubation, $1 \mathrm{ml}$ of a viscous overlay (1:1 $2 \times$ DMEM and 1.2\% methylcellulose) was added to each well. Plates were incubated for $4 \mathrm{~d}$. After fixation, staining and washing, plates were dried and plaques counted. Known neutralizing and non-neutralizing antibodies/sera are included in this assay as positive and negative controls, respectively. Plaques from each dilution of each sample were counted and data are reported as the concentration at which $50 \%$ of input virus was neutralized.

Pseudovirus neutralization assay. SARS-CoV-2 neutralization was assessed with Spike-pseudotyped viruses in 293T/ACE-2 cells as a function of reductions in luciferase (Luc) reporter activity. The 293T/ACE-2 cells were kindly provided by M. Farzan and H. Mu at Scripps. Cells were maintained in DMEM containing $10 \%$ FBS and $3 \mu \mathrm{g} \mathrm{ml}^{-1}$ of puromycin. An expression plasmid encoding codon-optimized full-length spike of the Wuhan-1 strain (VRC7480), was provided by B. Graham and K. Corbett at the Vaccine Research Center, National Institutes of Health. The D614G amino acid change was introduced into VRC7480 by site-directed mutagenesis using the QuikChange Lightning Site-Directed Mutagenesis Kit from Agilent Technologies (catalog no. 210518). The mutation was confirmed by full-length spike gene sequencing. Pseudovirions were produced in human embryonic kidney 293T/17 cells (ATCC catalog no. CRL-11268) by transfection using Fugene 6 (Promega catalog no. E2692) and a combination of spike plasmid, lentiviral backbone plasmid (pCMV $\Delta$ R8.2) and firefly Luc reporter gene plasmid $\left(\mathrm{pHR}^{\prime}\right.$ CMV Luc) ${ }^{23}$ in a 1:17:17 ratio. Transfections were allowed to proceed for $16-20 \mathrm{~h}$ at $37^{\circ} \mathrm{C}$. Medium was removed, monolayers rinsed with growth medium, and $15 \mathrm{ml}$ of fresh growth medium added. Pseudovirus-containing culture medium was collected after an additional $2 \mathrm{~d}$ of incubation, and was clarified of cells by low-speed centrifugation and $0.45-\mu \mathrm{m}$ micrometer filtration and stored in aliquots at $-80^{\circ} \mathrm{C}$. $\mathrm{TCID}_{50}$ assays were performed on thawed aliquots to determine the infectious dose for neutralization assays (relative luminescence units (RLU) 500$1,000 \times$ background; the background usually averages 50-100 RLU).

For neutralization, a pretitrated dose of virus was incubated with eight serial three- or fivefold dilutions of serum samples or mAbs in duplicate in a total volume of $150 \mu \mathrm{l}$ for $1 \mathrm{~h}$ at $37^{\circ} \mathrm{C}$ in 96-well flat-bottom poly-L-lysine-coated culture plates (Corning Biocoat). Cells were suspended using TrypLE express enzyme solution (Thermo Fisher Scientific) and immediately added to all wells (10,000 cells in $100 \mu \mathrm{l}$ of growth medium per well). One set of eight control wells received cells plus virus (virus control) and another set of eight wells received cells only (background control). After 66-72 h of incubation, medium was removed by gentle aspiration and $30 \mu \mathrm{l}$ of Promega $1 \times$ lysis buffer was added to all wells. After a $10-$ min incubation at room temperature, $100 \mu \mathrm{l}$ of Bright-Glo luciferase reagent was added to all wells. After 1-2 min, $110 \mu \mathrm{l}$ of the cell lysate was transferred to a black/white plate (PerkinElmer). Luminescence was measured using a PerkinElmer Life Sciences, Model Victor2 luminometer. Neutralization titers are the serum dilution (inhibitory doses of 50 or $80 \%$, ID $50 / \mathrm{ID} 80$ ) or mAb concentration (inhibitory concentrations of 50 or $80 \%$, $\mathrm{IC}_{50} / \mathrm{IC}_{80}$ ) at which RLU were reduced by 50 and $80 \%$ compared to virus control wells after subtraction of background RLU. Maximum percentage inhibition is the percentage neutralization at the lowest serum dilution or highest $\mathrm{mAb}$ concentration tested. Serum samples were heat-inactivated for $30 \mathrm{~min}$ at $56^{\circ} \mathrm{C}$ before assay.

SPR. Antibody binding to SARS-CoV-2 spike constructs was assessed using SPR on a Biacore T-200 (GE Healthcare) with HBS buffer with 3 mM EDTA and $0.05 \%$ 
surfactant P-20 added. For the data presented in Fig. $1 \mathrm{~d}$ and Extended Data Figs. 2 and 3 , spike samples that were incubated at 4,22 and $37^{\circ} \mathrm{C}$ for 1 week were held in the sample chamber at 8,22 and $37^{\circ} \mathrm{C}$, respectively, during the SPR run. The flow cell was maintained at $25^{\circ} \mathrm{C}$, thus all binding assays were performed at $25^{\circ} \mathrm{C}$. Antibodies captured on a CM5 chip coated with amine-coupled human Anti-Fc (8000RU) were assayed by SARS-CoV- 2 spike at $200 \mathrm{nM}$. The surface was regenerated between injections with $3 \mathrm{M} \mathrm{MgCl}_{2}$ solution for $10 \mathrm{~s}$ at $100 \mu \mathrm{min}^{-1}$. Sensorgram data were analyzed using the BiaEvaluation software (GE Healthcare).

Cryo-EM. Purified SARS-CoV-2 spike preparations were diluted to a concentration of roughly $1 \mathrm{mg} \mathrm{ml}^{-1}$ in $2 \mathrm{mM}$ Tris $\mathrm{pH} 8.0,200 \mathrm{mM} \mathrm{NaCl}$ and $0.02 \%$ $\mathrm{NaN}_{3}$. A 2.5- $\mu$ l drop of protein was deposited on a CF-1.2/1.3 grid that had been glow discharged for $30 \mathrm{~s}$ in a PELCO easiGlow Glow Discharge Cleaning System. After a $30 \mathrm{~s}$ incubation in $>95 \%$ humidity, excess protein was blotted away for $2.5 \mathrm{~s}$ before being plunge frozen into liquid ethane using a Leica EM GP2 plunge freezer (Leica Microsystems). Frozen grids were imaged in a Titan Krios (Thermo Fisher) equipped with a K3 detector (Gatan).

Reporting Summary. Further information on research design is available in the Nature Research Reporting Summary linked to this article.

\section{Data availability}

The NSEM reconstruction for rS2d-HexaPro has been deposited in the Electron Microscopy Data Bank with accession code EMD-22934. NSEM reconstructions for the $2 \mathrm{P}$ spike after 1-week cold storage followed by 3 -h recovery at $37^{\circ} \mathrm{C}$ are deposited with accession codes EMD-22967 and EMD-22968 for the 3-RBD-down and 1-RBD-up states, respectively.

\section{References}

17. Scheres, S. H. W. in Methods in Enzymology Vol. 579 (ed. Crowther, R. A.) 125-157 (Academic Press, 2016).

18. Liao, H. X. et al. Co-evolution of a broadly neutralizing HIV-1 antibody and founder virus. Nature 496, 469-476 (2013).

19. Alam, S. M. et al. Mimicry of an HIV broadly neutralizing antibody epitope with a synthetic glycopeptide. Sci. Transl. Med. 9, https://doi.org/10.1126/ scitranslmed.aai7521 (2017).

20. Bonsignori, M. et al. Staged induction of HIV-1 glycan-dependent broadly neutralizing antibodies. Sci. Trans. Med. 9, https://doi.org/10.1126/ scitranslmed.aai7514 (2017).

21. Berry, J. D. et al. Development and characterisation of neutralising monoclonal antibody to the SARS-coronavirus. J. Virol. Methods 120, 87-96 (2004).

22. Xie, X. et al. An infectious cDNA clone of SARS-CoV-2. Cell Host Microbe 27, 841-848.e3 (2020)

23. Naldini, L. et al. In vivo gene delivery and stable transduction of nondividing cells by a lentiviral vector. Science 272, 263-267 (1996).

\section{Acknowledgements}

This work was supported by National Institutes of Health, National Institute of Allergy and Infectious Diseases extramural project grant nos. R01 AI145687 (P.A.) and AI058607
(G.D.S.), funding from the Department of Defense HR0011-17-2-0069 (G.D.S.) and a contract from the State of North Carolina Pandemic Recovery Office through funds from the Coronavirus Aid, Relief and Economic Security Act (B.F.H.). We thank J. McLellan for providing the 2P S plasmid. This work used the DSC platform supported by the Duke Consortia for HIV/AIDS Vaccine Development and the Titan Krios microscope in the Duke University Shared Materials Instrumentation Facility, a member of the North Carolina Research Triangle Nanotechnology Network, which is supported by the National Science Foundation (award no. ECCS-2025064) as part of the National Nanotechnology Coordinated Infrastructure. The following reagent was deposited by the Centers for Disease Control and Prevention and obtained through BEI Resources, NIAID, NIH: SARS-Related Coronavirus 2, Isolate USA-WA1/2020, NR-52281. Microneutralization and PRNT assays were performed in the Virology Unit of the Duke Regional Biocontainment Laboratory, which received partial support for construction from the NIH NIAID (grant no. UC6-AI058607).

\section{Author contributions}

R.J.E., K. Mansouri., V.S. and P.A. discovered the effect of storage temperature on spike stability. R.J.E. led NSEM studies and established quantitative metrics for spike QC. K. Mansouri collected NSEM data and performed analyses. V.S. purified proteins and performed thermostability measurements. K. Manne purified proteins, performed SPR assays, prepared samples for NSEM, Tycho, ELISA, SPR and DSC measurements, and coordinated the study between the different research teams. B.W. performed DSC measurements. R.P., M.D., J.S. and W.W. performed ELISA assays. D.L., X.L., K.O.S. and G.D.S. isolated antibodies from convalescent patient. S.M.C.G. and K.J. purified proteins and performed thermostability assays. M.K purified proteins. Z.M. performed binding measurements. T.H.O., D.M. and G.D.S. performed neutralization assays. R.H. initiated the DSC experiments and provided the rS2d, 2P-N234A and 2P-N165A constructs before publication. S.M.A. supervised the DSC experiments. B.F.H. supervised ELISA experiments and antibody isolation. P.A. oversaw and led the study and cowrote the paper with R.J.E. and V.S. All authors reviewed and commented on the manuscript

\section{Competing interests}

The authors declare no competing interests.

\section{Additional information}

Extended data is available for this paper at https://doi.org/10.1038/s41594-020-00547-5.

Supplementary information is available for this paper at https://doi.org/10.1038/ s41594-020-00547-5.

Correspondence and requests for materials should be addressed to R.J.E. or P.A. Peer review information Nature Structural \& Molecular Biology thanks the anonymous reviewers for their contribution to the peer review of this work. Peer reviewer reports are available. Inês Chen was the primary editor on this article and managed its editorial process and peer review in collaboration with the rest of the editorial team.

Reprints and permissions information is available at www.nature.com/reprints. 
a

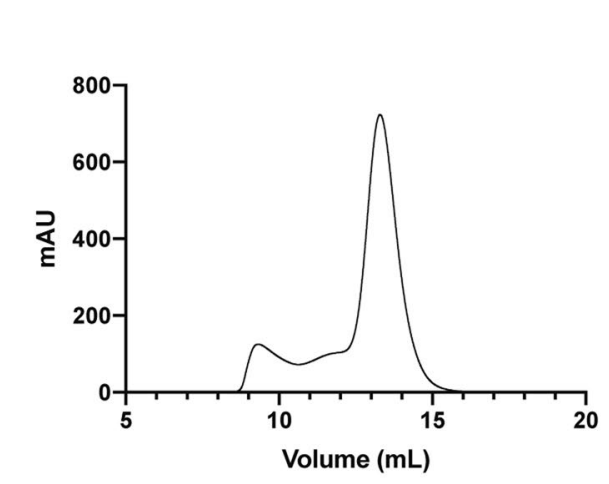

b

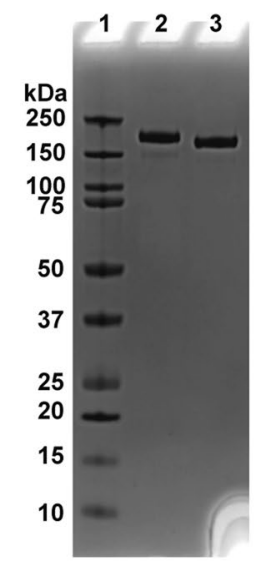

c

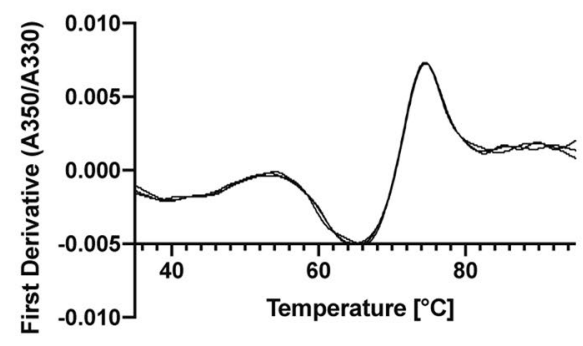

d

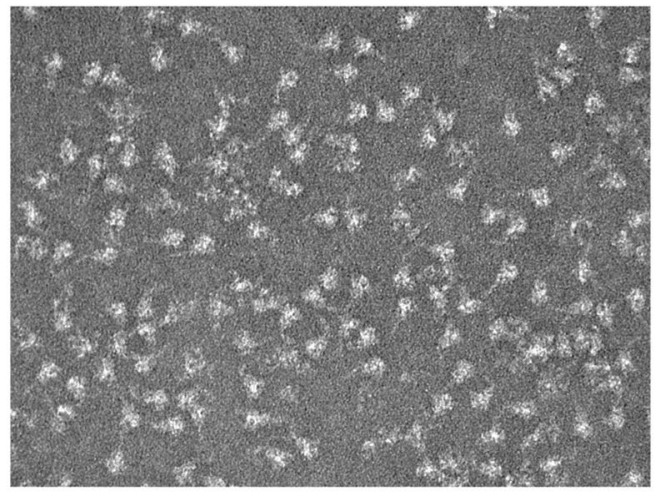

e

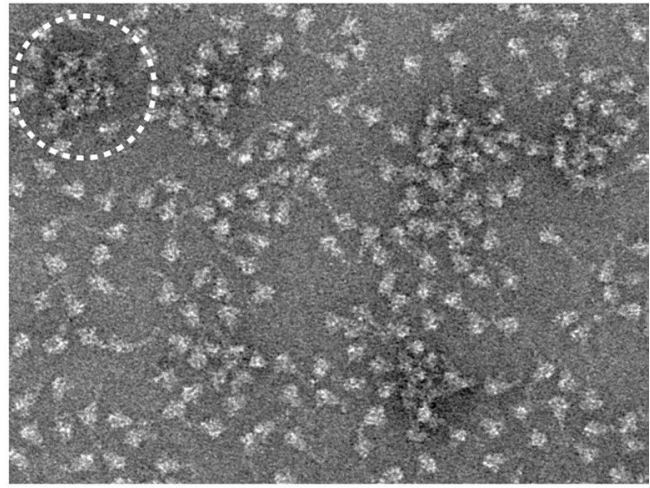

$100 \mathrm{~nm}$

Extended Data Fig. 1 | SDS-PAGE and NSEM analysis of SARS-CoV-2 2P S ectodomain incubated at different temperatures. (a) SEC profile, (b) SDS-PAGE analysis (lane 1= molecular weight marker, lane $2=$ reducing conditions, lane $3=$ non-reducing conditions) and (c) DSF profile of a freshly purified sample of $2 \mathrm{PS}$. (d) NSEM micrograph of $2 \mathrm{PS}$ after storage at $22^{\circ} \mathrm{C}$ for one week. (e) NSEM micrograph of $2 \mathrm{P} \mathrm{S}$ after storage at $4{ }^{\circ} \mathrm{C}$ for one week followed by recovery at $37^{\circ} \mathrm{C}$ for 6 days. The circle indicates spike aggregation visible in the micrograph. 
a

\section{CR3022 binding}
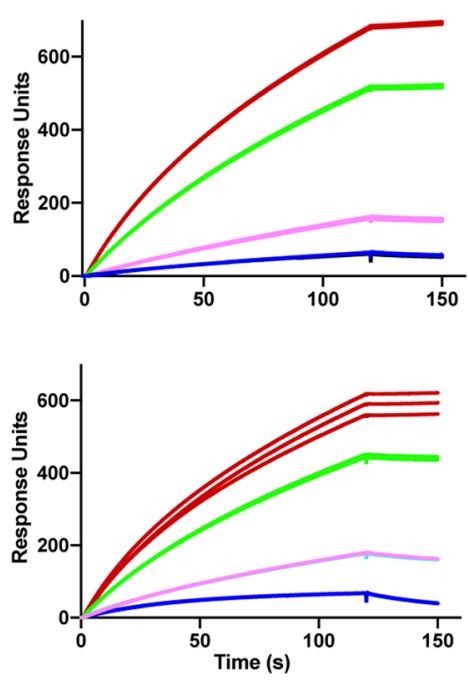

b

\section{ACE-2 binding}

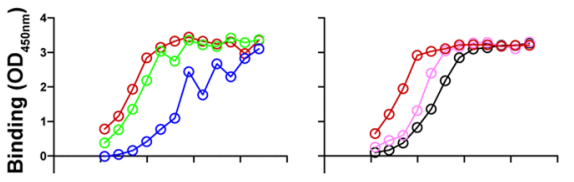

CR3022 binding

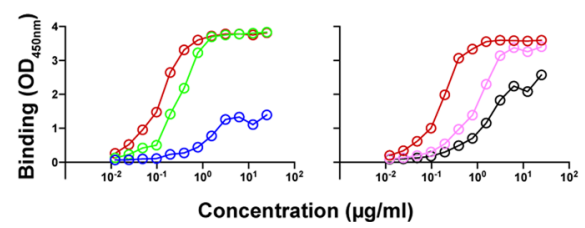

2G12 binding
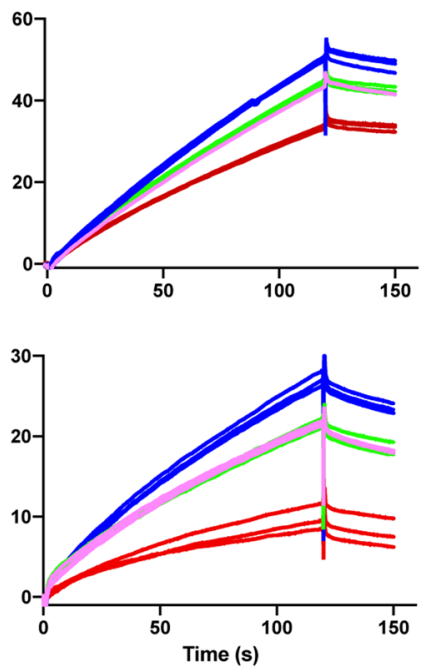

CR3022 binding
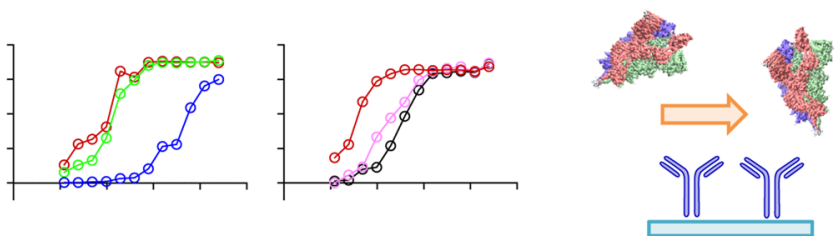

\section{G12 binding}

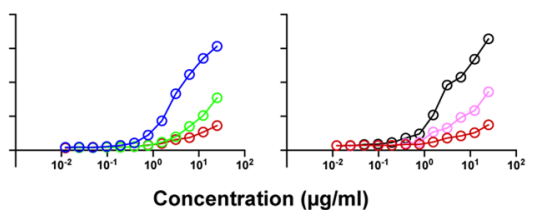

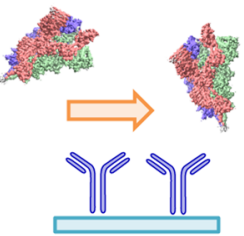

$37^{\circ} \mathrm{C}, 7 \mathrm{~d}$

$22^{\circ} \mathrm{C}, 7 \mathrm{~d}$

$4^{\circ} \mathrm{C}, 7 \mathrm{~d}$

$4^{\circ} \mathrm{C}, 7 \mathrm{~d} \longrightarrow 37^{\circ} \mathrm{C}$

Extended Data Fig. 2 | Changes in antigenicity of SARS-CoV-2 2PS ectodomain incubated at different temperatures. (a) Antibody CR3022 IgG (left) and $2 \mathrm{G} 12$ lgG (right) binding to two independently purified lots of 2P spike stored at different temperatures measured by SPR. Data for spike samples measured after a 1-week incubation at 37,22 , and $4^{\circ} \mathrm{C}$, are shown in blue, green, and red respectively; sample stored 1 week at $4{ }^{\circ} \mathrm{C}$ and then incubated for 6 hours at $37^{\circ} \mathrm{C}$ shown in cyan. During the SPR run the sample chamber was maintained at temperatures of $37^{\circ} \mathrm{C}, 22^{\circ} \mathrm{C}$ and $8{ }^{\circ} \mathrm{C}$, for the $37^{\circ} \mathrm{C}, 22^{\circ} \mathrm{C}$ and $4{ }^{\circ} \mathrm{C}$ incubated samples, respectively. The binding experiments were carried out at $25^{\circ} \mathrm{C}$. The bar graphs in Fig. $1 \mathrm{~d}$ show data from Lot \# $025 \mathrm{MFK}$. The schematics shows the assay format. (b) ELISA binding profiles showing binding of ACE-2 receptor ectodomain, RBD-directed antibody CR3022, and S2-directed, glycan-reactive antibody, 2G12. The same color scheme was used as for the SPR experiment. The black line indicates a freshly purified 2P spike sample that was flash frozen, then thawed and incubated for $20 \mathrm{~min}$ at $37^{\circ} \mathrm{C}$. The schematics show the ELISA format. The measurements in the top row were done in a format where antibody was coated on the plate and the measurements in the bottom row were done in a format where spike was captured on a strep-coated plate (see Methods). 
a

DH1179

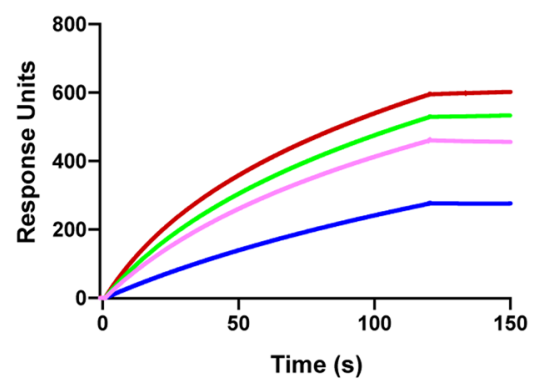

DH1189.1

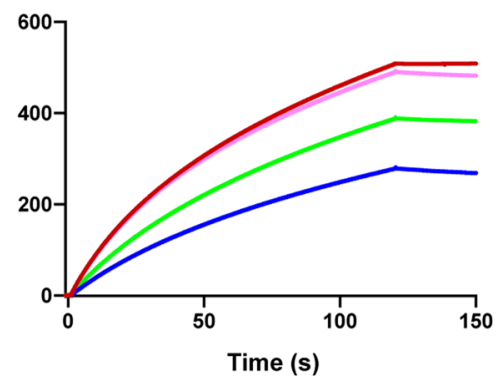

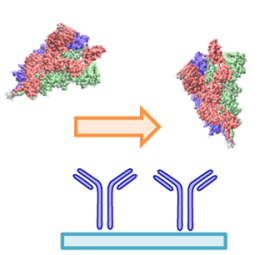

b

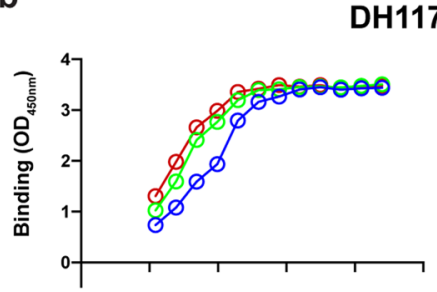

DH1179 binding

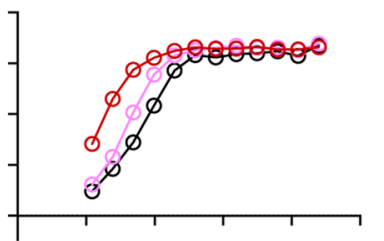

DH1189.1 binding
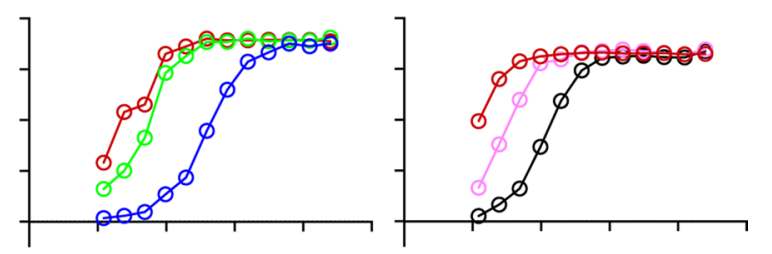

CH65 binding
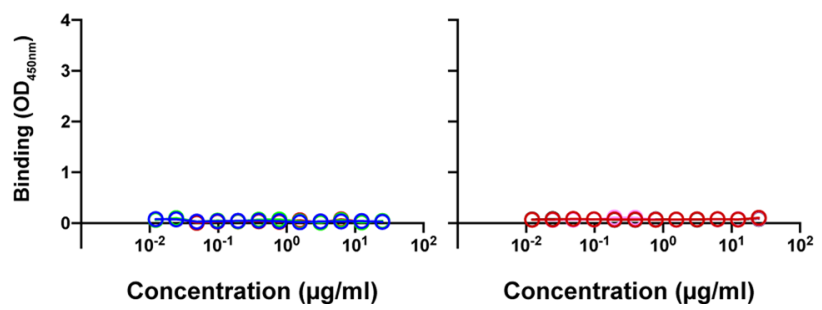

$37^{\circ} \mathrm{C}, 7 \mathrm{~d}$

$22^{\circ} \mathrm{C}, 7 \mathrm{~d}$

$4{ }^{\circ} \mathrm{C}, 7 \mathrm{~d}$

$4{ }^{\circ} \mathrm{C}, 7 \mathrm{~d} \longrightarrow 37^{\circ} \mathrm{C}$

Extended Data Fig. 3 | Antigenic response of SARS-CoV-2 2P S ectodomain incubated at different temperatures to antibodies elicited from convalescent patient sera. (a) SPR binding profiles showing binding of (left) RBD-directed antibody, DH1179 and (right) S2-directed antibody, DH1189.1 to spike samples incubated for 1 week at either $37^{\circ} \mathrm{C}$ (blue), $22^{\circ} \mathrm{C}$ (green) or $4{ }^{\circ} \mathrm{C}$ (red). Binding to spike sample first incubated at $4{ }^{\circ} \mathrm{C}$ for 1 week, then moved to $37^{\circ} \mathrm{C}$ for 3 hours prior to the experiment is shown in cyan. (b) ELISA binding profiles showing binding of RBD-directed antibody, DH1179, S2-directed antibody, DH1189.1, and influenza HA-directed antibody CH65 (control) to spike samples incubated at different temperatures (same color scheme as in panel a). The black line indicates a freshly purified 2P S sample that was flash-frozen, then thawed and incubated for 20 min at $37^{\circ} \mathrm{C}$. 


\section{Reporting Summary}

Nature Research wishes to improve the reproducibility of the work that we publish. This form provides structure for consistency and transparency in reporting. For further information on Nature Research policies, see our Editorial Policies and the Editorial Policy Checklist.

\section{Statistics}

For all statistical analyses, confirm that the following items are present in the figure legend, table legend, main text, or Methods section.

$\mathrm{n} / \mathrm{a}$ |Confirmed

$\bigotimes$ The exact sample size $(n)$ for each experimental group/condition, given as a discrete number and unit of measurement

$\square$ A statement on whether measurements were taken from distinct samples or whether the same sample was measured repeatedly

X The statistical test(s) used AND whether they are one- or two-sided

Xnly common tests should be described solely by name; describe more complex techniques in the Methods section.

$\triangle \square$ A description of all covariates tested

Х $\square$ A description of any assumptions or corrections, such as tests of normality and adjustment for multiple comparisons

$\triangle$ A full description of the statistical parameters including central tendency (e.g. means) or other basic estimates (e.g. regression coefficient)

X AND variation (e.g. standard deviation) or associated estimates of uncertainty (e.g. confidence intervals)

$X$ For null hypothesis testing, the test statistic (e.g. $F, t, r$ ) with confidence intervals, effect sizes, degrees of freedom and $P$ value noted

Х Give $P$ values as exact values whenever suitable.

Х $\square$ For Bayesian analysis, information on the choice of priors and Markov chain Monte Carlo settings

Х $\square$ For hierarchical and complex designs, identification of the appropriate level for tests and full reporting of outcomes

Х $\square$ Estimates of effect sizes (e.g. Cohen's $d$, Pearson's $r$ ), indicating how they were calculated

Our web collection on statistics for biologists contains articles on many of the points above.

\section{Software and code}

Policy information about availability of computer code

Data collection Provide a description of all commercial, open source and custom code used to collect the data in this study, specifying the version used OR state that no software was used.

Data analysis Pymol, UCSF Chimera, Relion, Biacore T200 Evaluation Software

For manuscripts utilizing custom algorithms or software that are central to the research but not yet described in published literature, software must be made available to editors and

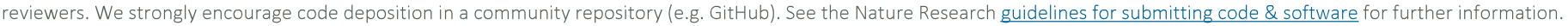

\section{Data}

Policy information about availability of data

All manuscripts must include a data availability statement. This statement should provide the following information, where applicable:

- Accession codes, unique identifiers, or web links for publicly available datasets

- A list of figures that have associated raw data

- A description of any restrictions on data availability

The NSEM reconstruction for rS2d-HexaPro has been deposited in the Electron Microscopy Data Bank with accession code EMD-22934. NSEM reconstructions for the $2 \mathrm{P}$ spike after 1-week cold storage followed by 3-hour recovery at $37^{\circ} \mathrm{C}$ are deposited with accession codes EMD-22967 and EMD-22968 for the 3-RBD-down and 1-RBD-up states, respectively. 


\section{Field-specific reporting}

Please select the one below that is the best fit for your research. If you are not sure, read the appropriate sections before making your selection.

$\bigotimes$ Life sciences

Behavioural \& social sciences

Ecological, evolutionary \& environmental sciences

For a reference copy of the document with all sections, see nature.com/documents/nr-reporting-summary-flat.pdf

\section{Life sciences study design}

All studies must disclose on these points even when the disclosure is negative.

Sample size n/a
Data exclusions n/a
Replication nandomization $\mathrm{n} / \mathrm{a}$
Blinding

\section{Reporting for specific materials, systems and methods}

We require information from authors about some types of materials, experimental systems and methods used in many studies. Here, indicate whether each material, system or method listed is relevant to your study. If you are not sure if a list item applies to your research, read the appropriate section before selecting a response.

\begin{tabular}{l|l} 
Materials \& experimental system \\
\hline $\mathrm{n} / \mathrm{a}$ & Involved in the study \\
\hline & $\bigotimes$ Antibodies \\
$\square$ & $\bigotimes$ Eukaryotic cell lines \\
$\square$ & $\square$ Palaeontology and archaeology \\
$\square$ & $\square$ Animals and other organisms \\
$\searrow$ & $\square$ Clinical data \\
$\searrow$ & $\square$ Dual use research of concern
\end{tabular}

Methods

$\mathrm{n} / \mathrm{a}$ Involved in the study

Х $\square$ ChIP-seq

Х $\square$ Flow cytometry

\ $\square$ MRI-based neuroimaging

\section{Antibodies}

\section{Antibodies used}

CR3022, 2G12, DH1179, DH1189.1

Validation

Cloned in-house and verified by DNA sequencing. Purified antibody validated using SDS-PAGE and binding to SARS-CoV-2 2PS by flow cytometry, SPR and ELISA.

\section{Eukaryotic cell lines}

Policy information about cell lines

Cell line source(s)

Freestyle 293 cells and CHO cells from Invitrogen was used to express S-protein trimers

Authentication

Manufacturer of the cell line provides authentication information on their website

Mycoplasma contamination

Mycoplasma contamination screening is described on the manufacturer's website

Commonly misidentified lines

(See ICLAC register) 